\title{
Numerical and experimental study on confinement in Y-shaped post wall branching waveguide
}

\author{
Hiroshi Maeda $^{\mathrm{a}, *}$, Huili Chen ${ }^{\mathrm{b}}$, Kazuya Tomiura ${ }^{\mathrm{b}}$ and Kiyotoshi Yasumoto ${ }^{\mathrm{c}}$ \\ ${ }^{a}$ Department of Information and Communication Engineering, Fukuoka Institute of Technology, \\ Higashi-Ku, Fukuoka, Japan \\ ${ }^{\mathrm{b}}$ Graduate School of Engineering, Fukuoka Institute of Technology, Higashi-Ku, Fukuoka, Japan \\ ${ }^{\mathrm{c}}$ Computer Science Laboratory, Fukuoka Institute of Technology, Higashi-Ku, Fukuoka, Japan
}

\begin{abstract}
Post wall waveguides consist of dielectric or metallic cylinders for microwave around $4 \mathrm{GHz}$ were experimentally and numerically investigated. The structure attracts interests for application of transmission circuit for three-dimensionally integrated planar circuit in millimeter wavelength. In straight waveguide with dielectric cylinders, confinement of microwave is poor, when the post wall was composed of a pair of single row of cylinders. The confinement was improved as increase of rows of post wall. In metallic cylinders, microwave was well confined even when a pair of single row of cylinders composed the waveguide. After confirming confinement of the electromagnetic field, Y-shaped branches of post wall waveguide consisted of dielectric cylinders for microwave were similarly investigated for dielectric rods. The confinement was also improved by increase of post wall up to 3 layers. These results are applicable for fundamental design and fabrication of integrated circuit for microwave and millimeter wave.
\end{abstract}

Keywords: CIP method, experiment, microwave, numerical analysis, post wall waveguide, Y-branch waveguide

\section{Introduction}

Post wall waveguides are attracting much attentions as transmission line for microwave and millimeter wave. The structure is quite simple such as periodic array of dielectric or metallic cylinders, but shows good confinement of microwaves. As well-known similar periodic structure, photonic crystal (PC) waveguide [1] has intensively studied as substance of conventional optical waveguides using total internal reflection. The PC structure has photonic band gap (PBG) or electromagnetic band gap (EBG) for limited range of frequency band and propagating direction. Authors' group has been investigated transmission characteristics of two dimensional photonic crystal waveguide by numerical method and confirmed the results by scale model in microwave frequency. The experiment by scale model $[2,3]$ is easily transformed to post wall waveguide, because both structures consist of periodic array of dielectric or metallic cylinders [4-7].

In this paper, basic transmission characteristics of straight waveguide and Y-shaped branch waveguide consisted of post wall structure, are reported. For the waveguide with dielectric cylinders, experimental

\footnotetext{
${ }^{*}$ Corresponding author: Hiroshi Maeda, Department of Information and Communication Engineering, Fukuoka Institute of Technology (FIT), 3-30-1 Wajiro-Higashi, Higashi-Ku, Fukuoka 811-0295, Japan. E-mail: hiroshi@ fit.ac.jp.
} 


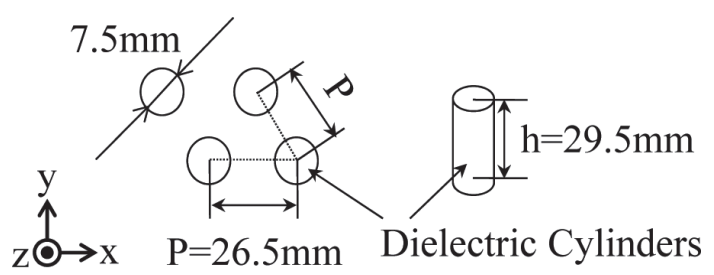

(a) Unit cell

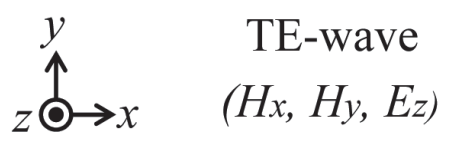

(b) TE polarized components

Fig. 1. Illustration of (a) unit cell of periodic triangular array and (b) polarization of electromagnetic field components.

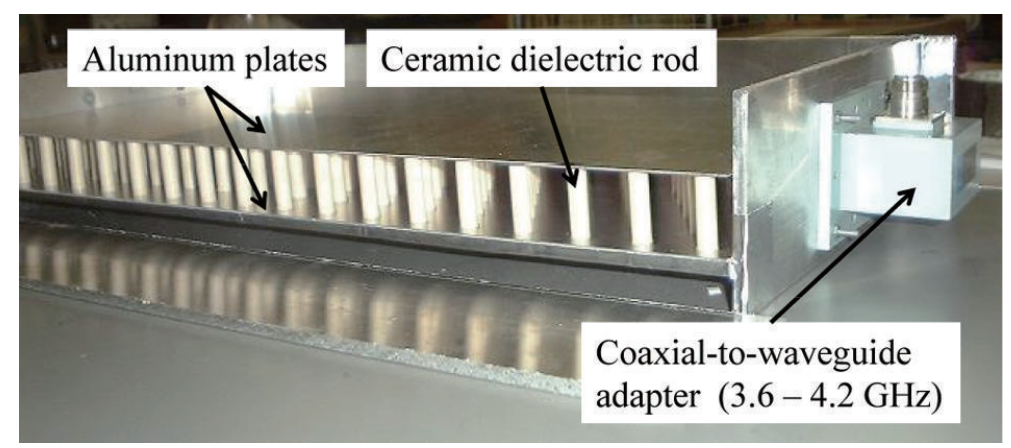

Fig. 2. Side view of a scale model of two dimensional photonic crystal structure. Through coaxial-to-waveguide adapter, microwave is excited and received.

results and numerical results corresponded with each other on the confinement. The basic design scheme for post wall waveguide by numerical and experimental approaches were established throughout this study. These results are applicable for design of microwave and optical integrated circuits as guiding structure can be composed of simple arrays of dielectric cylinders.

This paper is composed as followings. In Section 2, straight post wall waveguide is analyzed by experiment and numerical analysis. Setup of the experiment, measurements, and simulation results by the constrained interpolation profile (CIP) method are explained in each subsection. An important result for electromagnetic confinement in the waveguide was obtained. In Section 3, Y-shaped post array waveguides are investigated experimentally and numerically. Related to Section 2, we could reach an important fact on the confinement in branching waveguide. In Section 4, confinement of the electromagnetic wave is summarized. For general periodic structures, it is also mentioned that our experimental approach proposed here could be applicable.

\section{Straight post wall waveguide}

\subsection{Experimental setup for microwave measurement}

In Fig. 1(a), top view of unit triangular cell is illustrated, together with height of the cylinder. The coordinate axis is also shown in the figure. The longitudinal axis of the cylinder corresponds with polariza- 


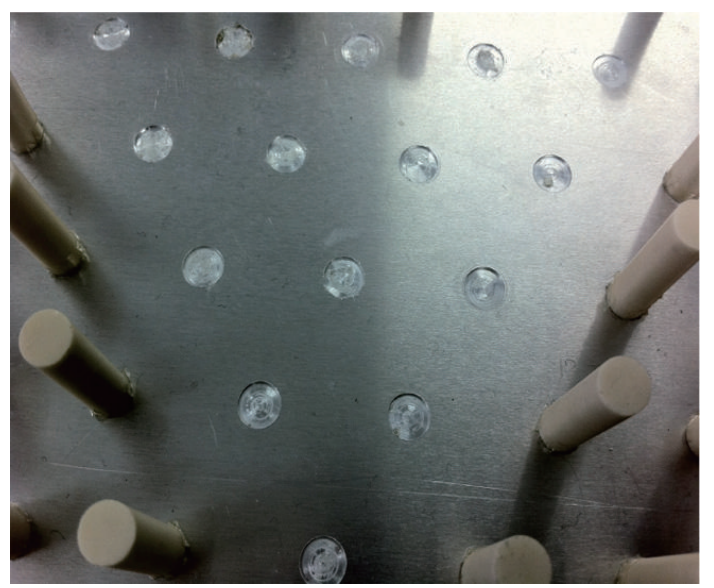

Fig. 3. Shallow periodic guide holes fabricated by numerically controlled milling machine.

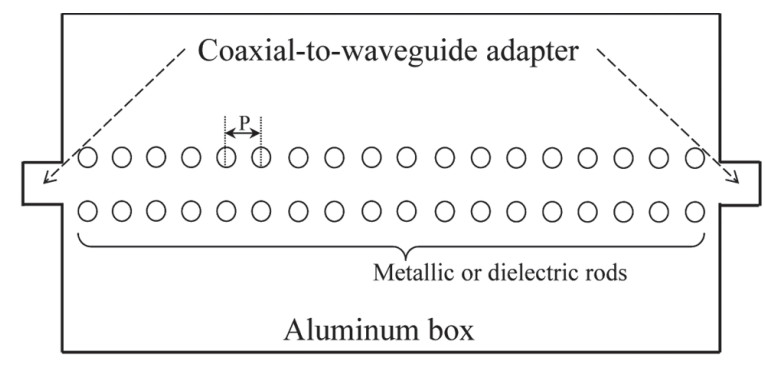

(a) Pair of single rows of cylinders

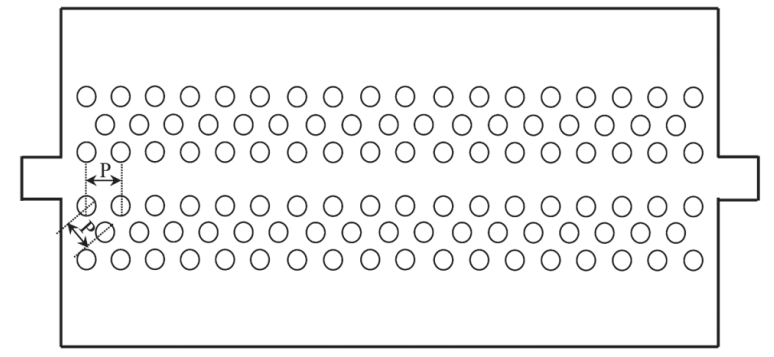

(b) Pair of 3 rows of cylinders

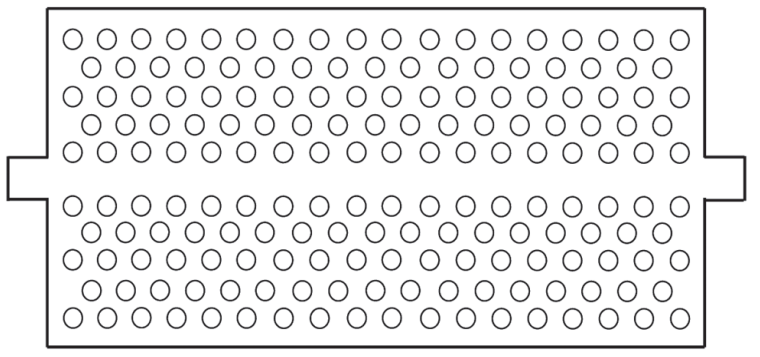

(c) Pair of 5 rows of cylinders

Fig. 4. Schematic of top view of post wall waveguides. 


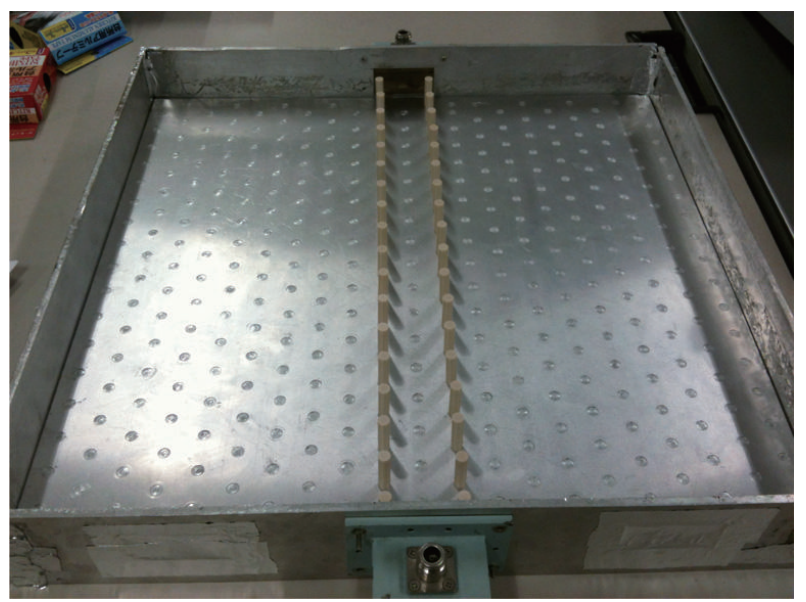

Fig. 5. Photograph of post wall waveguide consisted of a pair of single row of cylinders with ceramic rods. The waveguide is excited and received by coaxial-to-waveguide adapter.

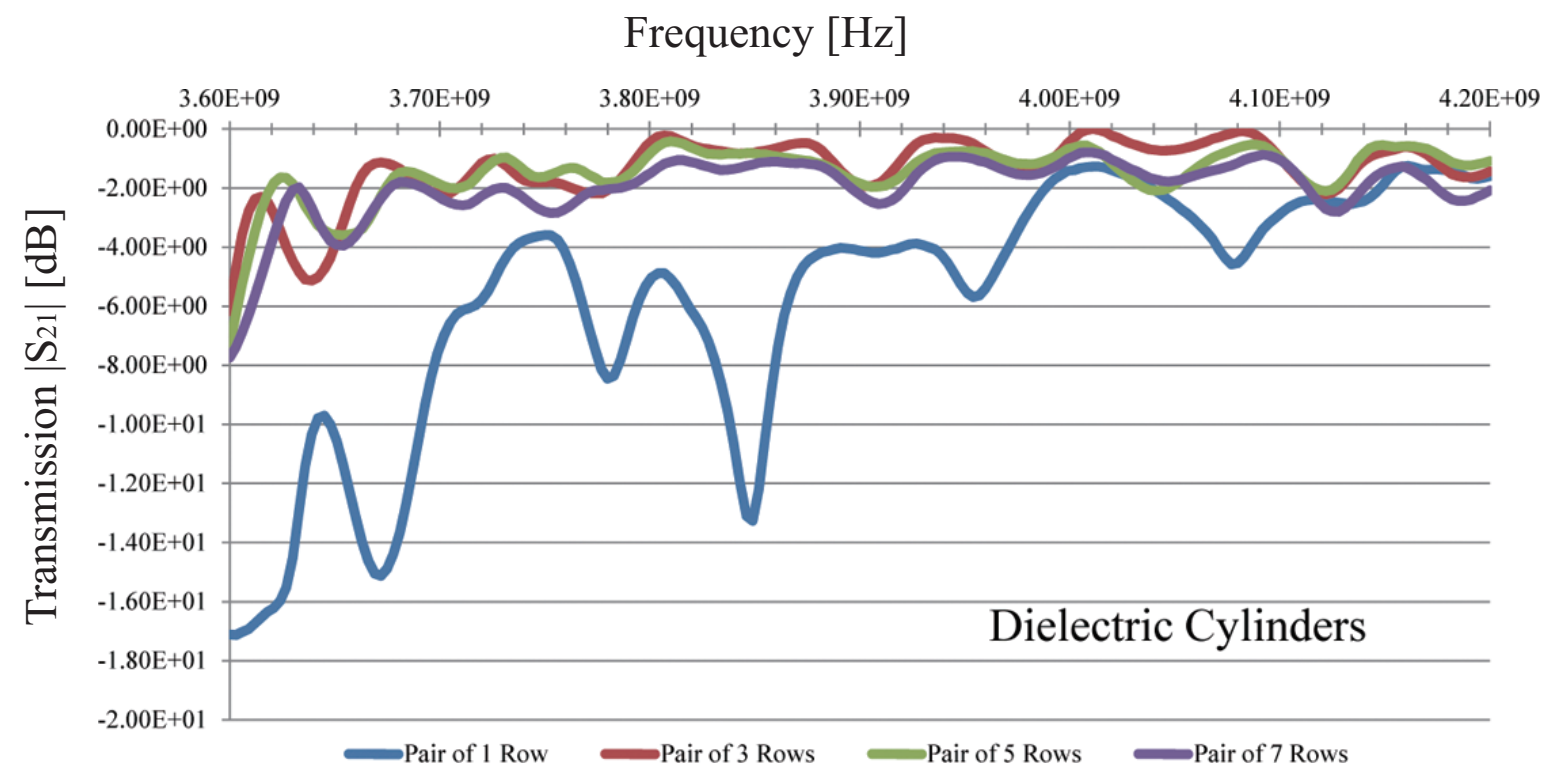

Fig. 6. Transmission Characteristics of post wall waveguide with ceramic cylinders. Measurement of single row is also plotted.

tion direction of electric field, as is shown in Fig. 1(b). Transverse electric (TE) mode with components $\left(H_{x}, H_{y}, E_{z}\right)$ is excited in the input waveguide adapter.

To demonstrate two dimensional experiment, we put periodic ceramic rods between two aluminum plates, as is depicted in Fig. 2. These aluminum plates were grounded to assume this structure is artificially two dimensional under 'principle of mirror image' in electromagnetic theory. Dielectric rods were put periodically by using shallow guide holes, which were fabricated by mechanical mill machine with precision order of 1 micrometer. Material of the dielectric rod is ceramic for dielectric post.

Measurements were done by using vector network analyzer Agilent Technologies E5071C. Coaxialto-waveguide adapters were used for input and output, while one of output ports of Y-shaped waveg- 


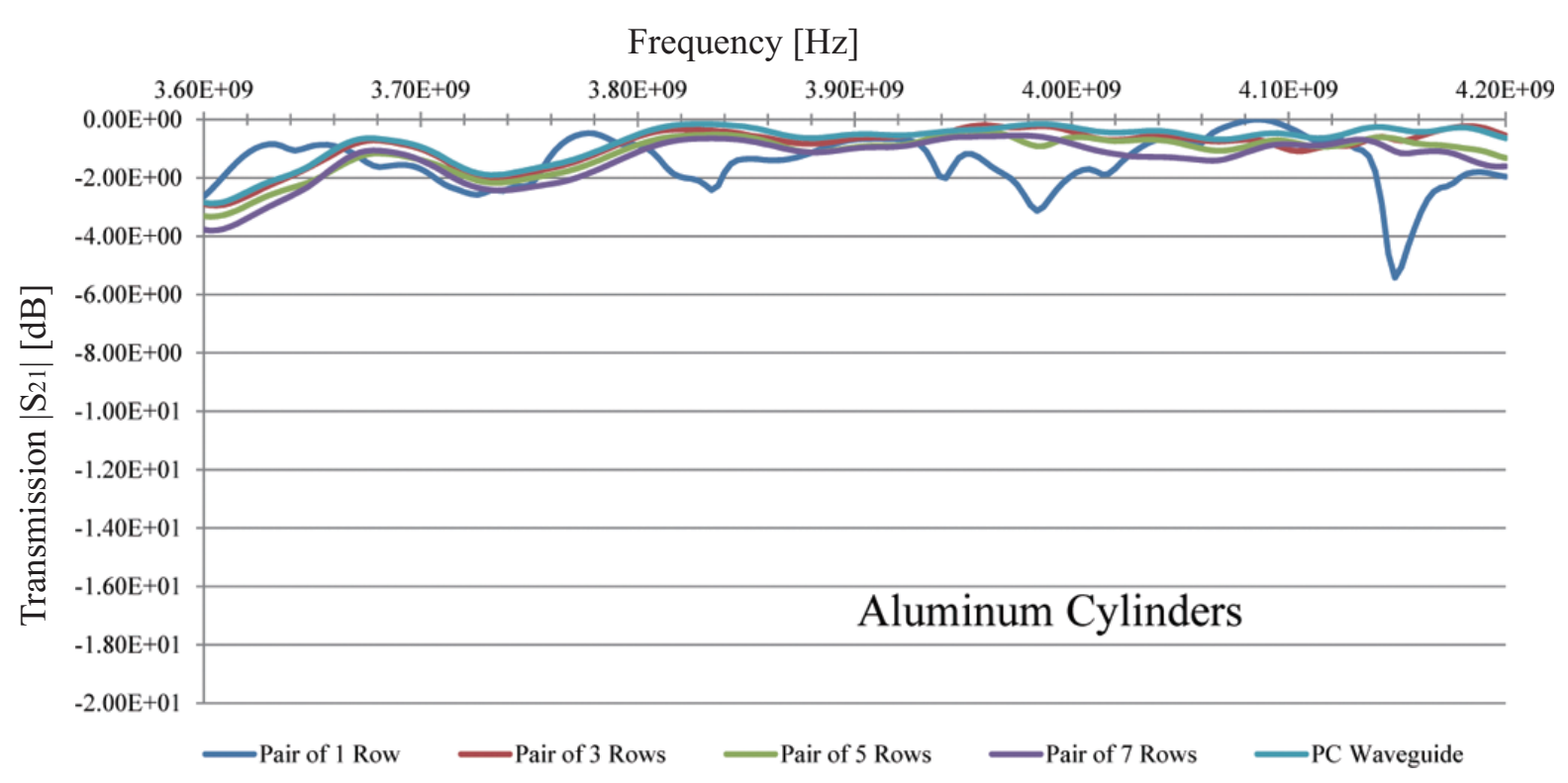

Fig. 7. Transmission Characteristics of post wall waveguide with aluminum cylinders.

uide which is not under measurement was terminated by anti-reflection adapter. Frequency range of the measurement is from $3.6 \mathrm{GHz}$ to $4.2 \mathrm{GHz}$, which corresponds to operation range of input and output waveguide adapters.

\subsection{Measurement of straight post wall waveguide}

Schematic illustrations of straight post wall waveguides were shown in Fig. 4. In Fig. 4(a), waveguide is consisted of a pair of single row of posts. In Figs 4(b) and (c), number of surrounding rows were increased to 3 and 5 rows respectively.

In Fig. 5, photograph of post wall waveguide with a pair of single row of cylinders is shown. An covering plate over the waveguide is removed for taking a photograph. Material of the posts is ceramic, which was made of Kyocera Co. ltd, Japan with relative dielectric constant $\varepsilon_{r}=36.0$ at $4 \mathrm{GHz}$. This waveguide is excited and received by coaxial-to-waveguide adapters. Material of the metallic box is aluminum. When measurement was done, aluminum plate covers entire box to achieve the structure as two dimensional supposing 'principle of mirror image'.

In Figs 6 and 7, measured transmission characteristics of post wall waveguide are shown for dielectric post and metallic post, respectively. In Fig. 6, amount of transmission is small only when the waveguide is conposed of a pair of single row cylinders, as is depicted in Fig. 4(a). By increasing rows of surrounding posts, the transmissions are improved.

On the other hand, as shown in Fig. 7, metalic post wall waveguide confines microwave very well, even when a pair of single row of cylinders consists of waveguide. The confinement of $1,3,5$, and 7 rows of cylinders made no difference.

\subsection{Numerical analysis of straight post wall waveguide}

To confirm experimental results in previous section, we demonstrate numerical analysis by constrained interpolated profile (CIP) method. The CIP method was proposed by Yabe [8] and applied for electro- 


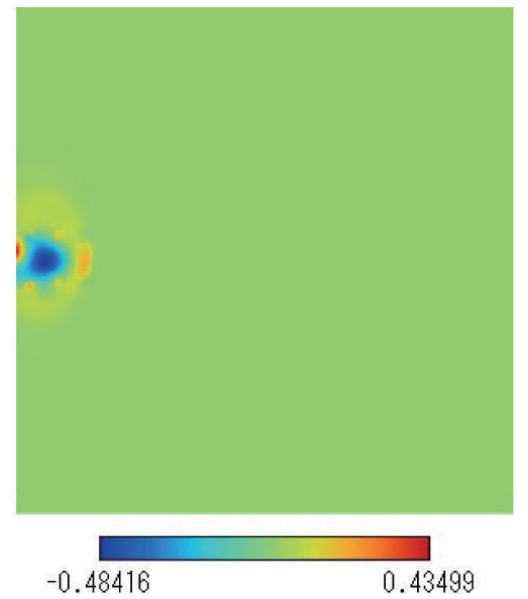

(a) $1000 \Delta \mathrm{t}$ for single rows

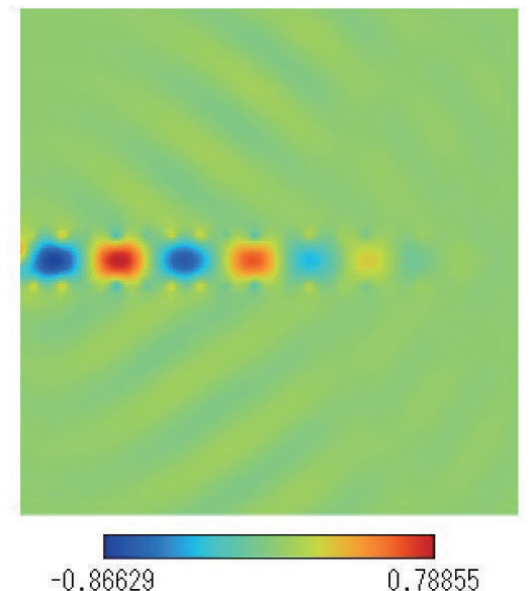

(b) $10000 \Delta t$ for single rows

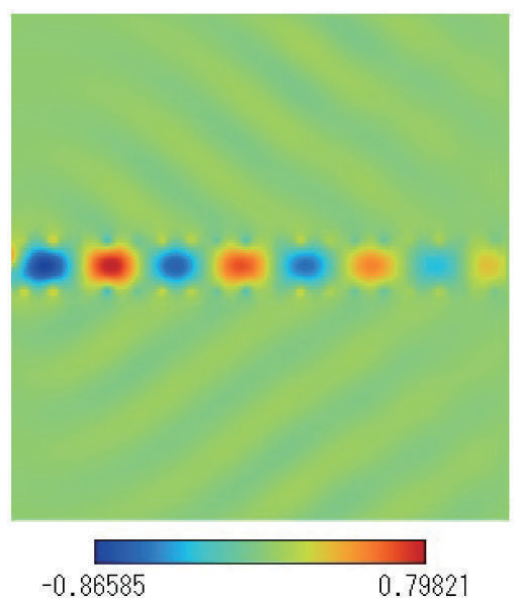

(c) $15000 \Delta \mathrm{t}$ for single rows

Fig. 8. Electric field profile in post wall waveguide for $f=4.0 \mathrm{GHz}$ with a pair of single row of cylinders by CIP method at typical time steps.

magnetic wave analysis by Okubo [9]. Advantage of CIP method compared with conventional finitedifference time-domain (FDTD) method [10] is superiority of accuracy of scattered wave by target with relatively high material constant from the background medium [11,12]. The superiority of accuracy of CIP method was confirmed for scattering problem of single dielectric cylinder, by comparing with analytical method based on Hertz potential [13,14].

In the following examples of two-dimensional analysis, space grid size $\Delta x=\Delta y=0.5 \mathrm{~mm}$ and time step $\Delta t=1.7 \times 10^{-13} \mathrm{sec}$ were used.

In Fig. 8, time evolution of electric field profile of TE mode in post wall waveguide with a pair of single row dielectric cylinders is shown for typical three time steps. As seen in Figs 8(b) and (c), the waveguide is leaky and radiated wave is observed.

To compare the confinement with different number of rows, electric field profile in waveguide with a pair of 3 rows and that with 5 rows are shown in Figs 9 and 10, respectively. It is shown that the confinement is very well even for a pair of 3 rods. These results agree with mesurement in Fig. 6 very well. 


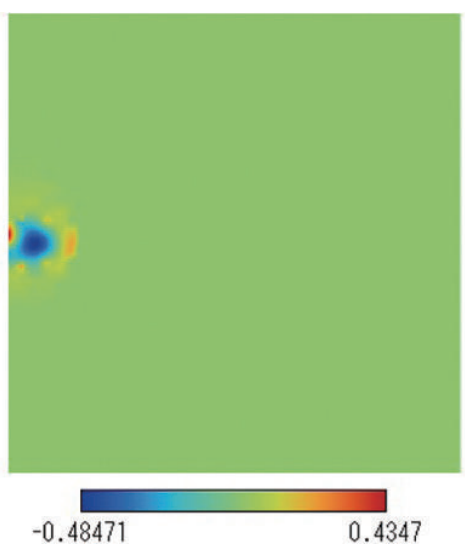

(a) $1000 \Delta \mathrm{t}$ for 3 rows

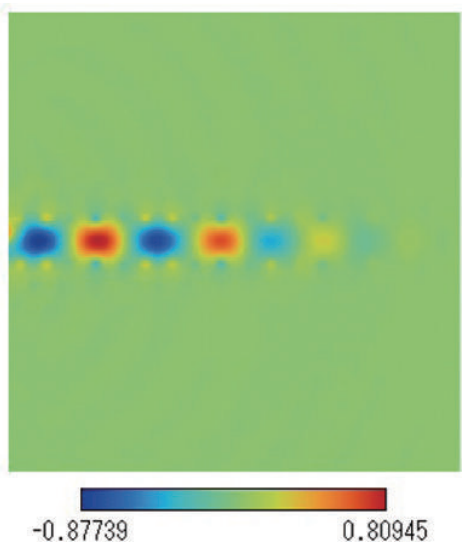

(b) $10000 \Delta \mathrm{t}$ for 3 rows

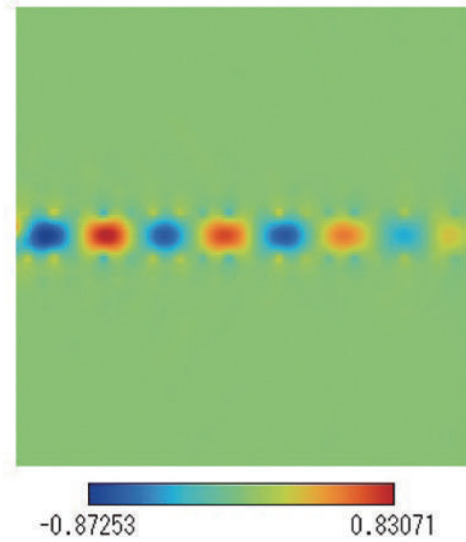

(c) $15000 \Delta \mathrm{t}$ for 3 rows

Fig. 9. Electric field profile in post wall waveguide for $f=4.0 \mathrm{GHz}$ with a pair of 3 rows of cylinders by CIP method.

\section{Measurement and simulation of post wall Y-shaped waveguide}

In Fig. 11(a), top view of post wall Y-shaped waveguide composed of a pair of periodic dielectric array with single layer is shown. The structure was fabricated by making use of the PC structure shown in Fig. 12(a) by removing unnecessary dielectric rods. In microwave measurement, radiated wave from post wall waveguide is expected. The radiation must be removed to observe isolated guided wave at output port. Therefore, electromagnetic wave absorber was put along with surrounding aluminum wall to eliminate reflected wave. Measurement results of the structure are shown in Fig. 11(b). The transmissions $S 21$ and $S 31$ by measurement are smaller than that of Fig. 12(b). Also, the reflections $S 11-2$ and $S 11-3$ are almost in the same magnitude with Fig. 12(b)

For implementation of this phenomena, numerical analysis by the CIP method were demonstrated. The parameters are same with previous section. Reflection from end of the calculated area is negligibly small in the CIP analysis [8]. Electric field profile of $E_{z}$ by numerical analysis is shown in Fig. 11(c) as a typical case after steady state at $20,000 \Delta t$ for input frequency $f=4.0 \mathrm{GHz}$. As is shown in the figure, radiated wave is obviously exists around branching point and along with waveguides after the branch. This means that post wall Y-shaped branch consisted of a pair of 1 layer periodic array has poor confinement characteristics. 


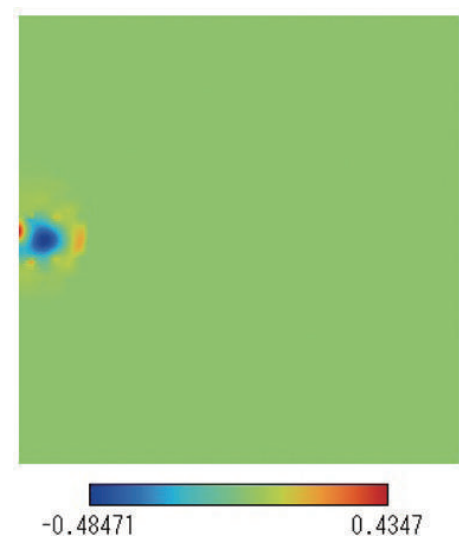

(a) $1000 \Delta t$ for 5 rows

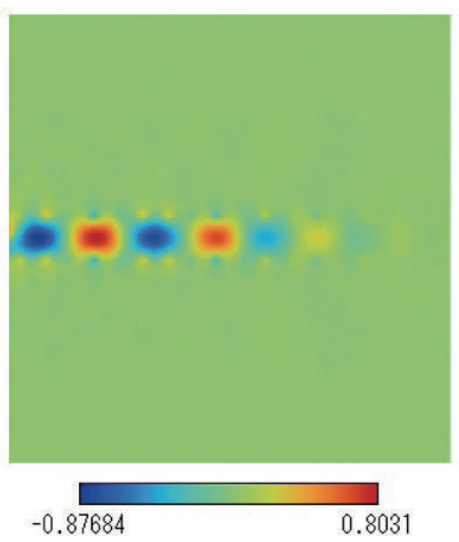

(b) $10000 \Delta \mathrm{t}$ for 5 rows

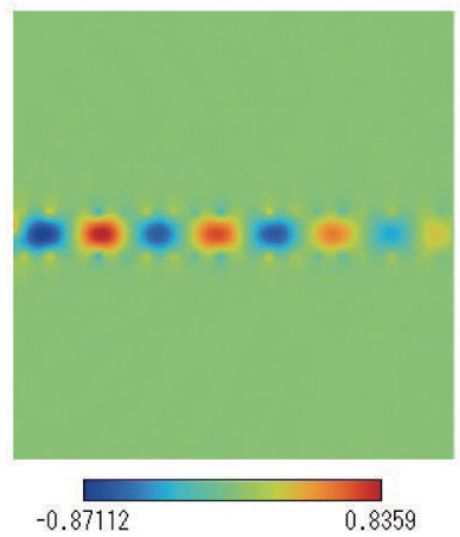

(c) $15000 \Delta \mathrm{t}$ for 5 rows

Fig. 10. Electric field profile in post wall waveguide for $f=4.0 \mathrm{GHz}$ with a pair of 5 rows of cylinders by CIP method.

In Fig. 13(a), top view of post wall waveguide composed by a pair of periodic dielectric array with 3 layers is shown. The structure was fabricated also by making use of the PC structure. Measurement results of the structure are shown in Fig. 13(b). The transmissions and reflections are designated in the same manner as Fig. 11. Simulation result by CIP method was also demonstrated for comparison. Electric field profile of $E_{z}$ is shown in Fig. 13(c) at the same time step as Fig. 11(c) for input frequency $f=4.0 \mathrm{GHz}$. From those figures, it was found that electric field profile is well confined and propagated all through the waveguides. For confinement and propagation of electromagnetic field in post wall Yshaped branch waveguide, a pair of periodic array with 3 layers are necessary.

\section{Conclusions}

In this paper, experimental and numerical results of post wall waveguide were shown. It was found that a pair of periodic walls with 3 layers are needed for confinement and propagation of electromagnetic field in dielectric posts. For metallic posts, even a pair of single layers of periodic array could confine the field.

This result is applicable for integrated microwave and millimeter wave circuit. By applying scaling rule, this circuit operates for optical frequency range. These results are applicable for PBG structure with 


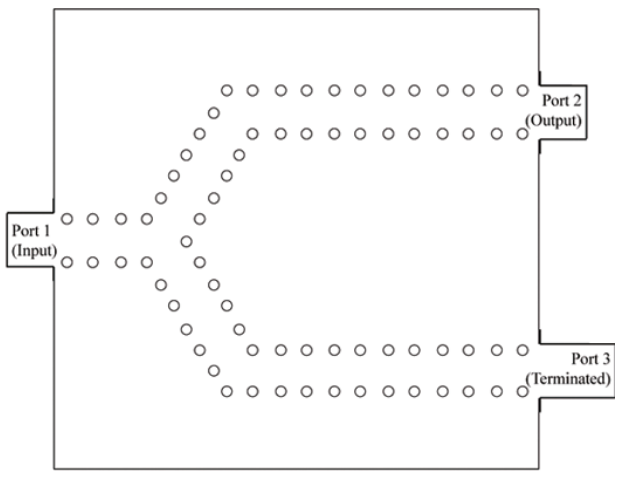

(a) Top view of post wall waveguide with 1 layer

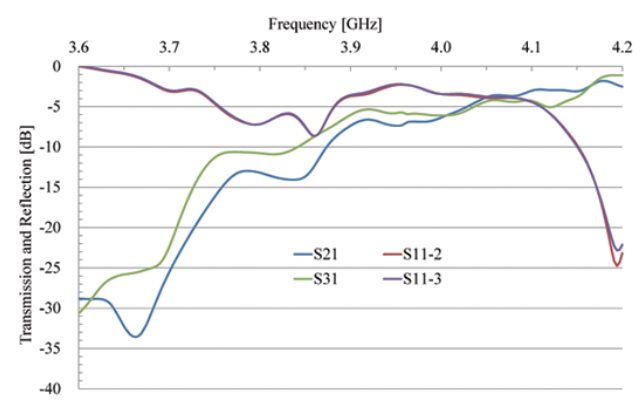

(b) Measurement results

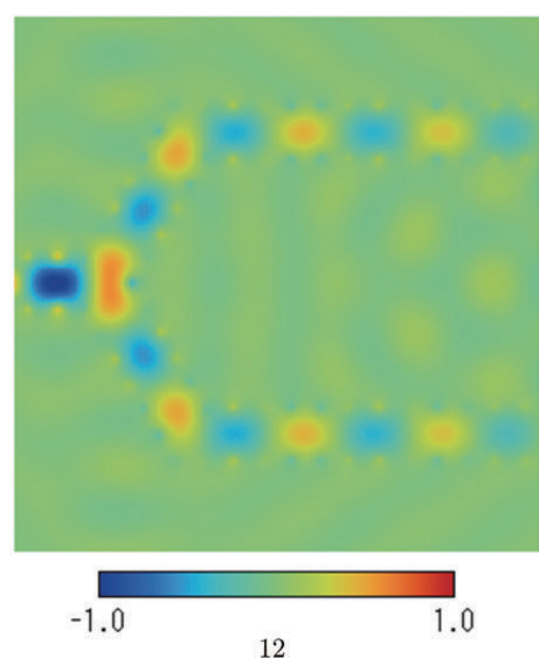

(c) Simulation result of Ez for $4.0 \mathrm{GHz}$

Fig. 11. Post wall waveguide with single layer of periodic array.

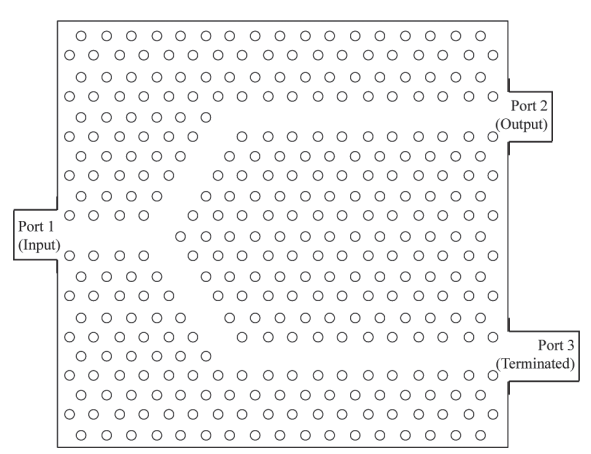

(a) Top view of two dimensional PC

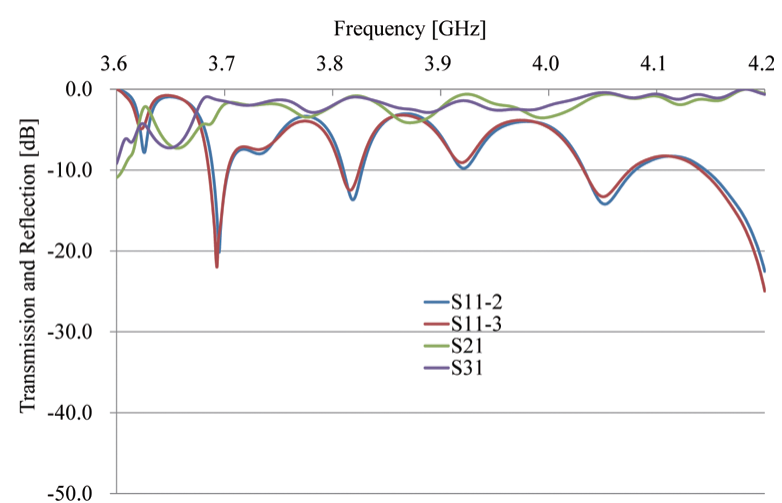

(b) Measurement results

Fig. 12. Photonic crystal(PC) Y-shaped waveguide as reference. (a) Top view of two dimensional PC waveguide, (b) Measured transmission (S21 and S31) and reflection (S11-2 and S11-3) characteristics. 


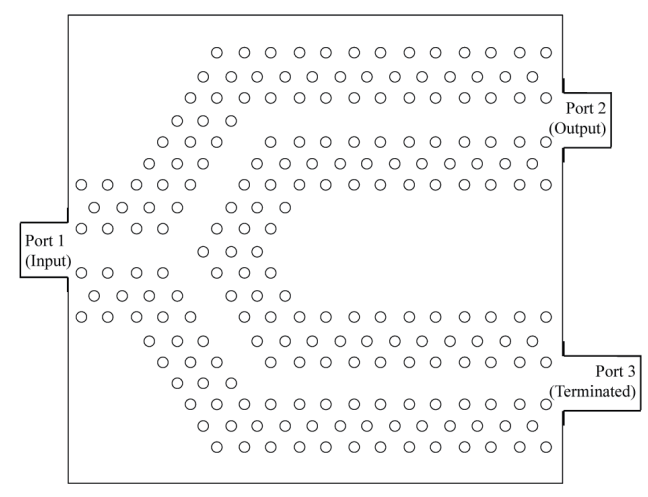

(a) Top view of post wall waveguide with 3 layers

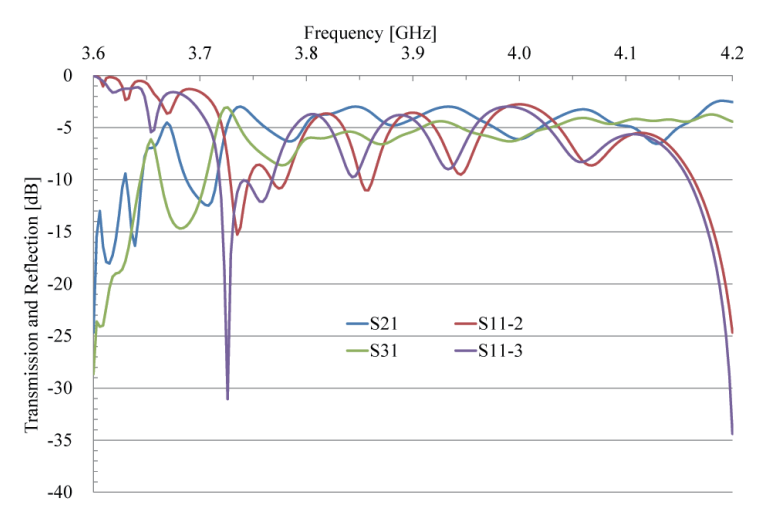

(b) Measurement results

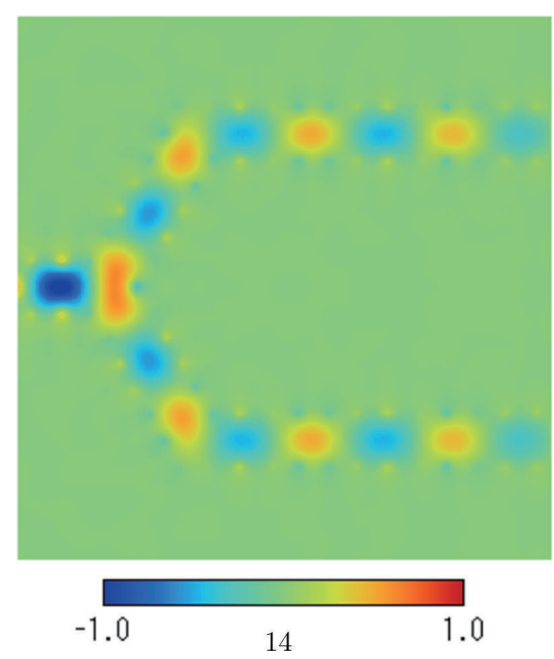

(c) Simulation result of Ez for $4.0 \mathrm{GHz}$

Fig. 13. Post wall waveguide with 3 layers of periodic array.

integrated transmission lines. Also, this investigation gives an approach for general design of microwave and optical integrated circuits, as those guiding structure can be composed of simple arrays of cylinders in which we analyzed in this work.

\section{Acknowledgments}

This work was financially supported by KAKENHI No. 24560430, Grant-in-Aid for Scientific Research (C) by Japan Society for the Promotion of Science(JSPS) in 2012. Authors express our thanks also to D. Kunitake, K. Kosugi, K. Satoh, M. Maruyama, T. Izumi, and Y. Yano for their cooperation with measurement of post wall waveguide by scale model as part of their graduation research.

\section{References}

[1] K. Yasumoto, ed., Electromagnetic Theory and Applications for Photonic Crystals, CRC PRESS, 2006. 
[2] B. Temelkuran and E. Ozbay, Experimental demonstration of photonic crystal based waveguides, Applied Physics Letters 74(4) (Jan. 1999), 486-488.

[3] M. Beaky et al., Two-dimensional photonic crystal Fabry-Perot resonators with lossy dielectrics, IEEE Transactions on Microwave Theory and Techniques 47(11) (Nov. 1999), 2085-2091.

[4] Y. Zhang, H. Terashima, D. Ogata and H. Maeda, Some Trials to Suppress Unnecessary Resonance in Photonic Crystal Cavity Structure, Proceedings of 6th International Conference on Broadband, Wireless Computing, Communication and Applications (BWCCA 2011), Oct. 2011, pp. 482-486.

[5] H. Maeda, H. Chen, K. Tomiura and T. Shinohara, Numerical and Experimental Study on Confinement of Microwave in Dielectric and Metallic Post Array Waveguide, Proceedings of The 2nd International Workshop on Information Networking and Wireless Communications (INWC 2012), in conjunction with The 15th International Conference on NetworkBased Information Systems (NBiS 2012), to be appeared in September, 2012.

[6] H. Maeda, Y. Zhang, H. Terashima and T. Shinohara, Hexagonal resonator in two dimensional photonic crystal structure, Proceedings of Third IEEE International Conference on Intelligent Networking and Collaborative Systems (IEEE INCOS 2011), Nov. 2011, pp. 537-540.

[7] H. Maeda, Y. Zhang, H. Terashima, T. Maruyama and S. Hironaka, Experimental Study on Four-Branching Waveguide in Two Dimensional Photonic Crystal Structure with Triangular Lattice by Scale Model around $4 \mathrm{GHz}$, Proceedings of 13th International Symposium on Microwave and Optical Technology (ISMOT-2011), June 2011, pp. 65-68.

[8] T. Yabe, X. Feng and T. Utsumi, The constrained interpolation profile method for multiphase analysis, Journal of Computational Physics 169 (2001), 556-593.

[9] K. Okubo and N. Takeuchi, Analysis of an electromagnetic field created by line current using constrained interpolation profile method, IEEE Transactions on Antennas and Propagation 55(1) (Jan. 2007), 111-119.

[10] A. Taflove, ed., Advances in Computational Electrodynamics - The Finite-Difference Time-Domain Method, Artech House Publishers, 1998.

[11] H. Maeda, H. Terashima, T. Maruyama and S. Hironaka, Numerical analysis of scattered electromagnetic wave by a dielectric cylinder with high contrast by CIP method, Proceedings of First International Workshop on Methods, Analysis and Protocols for Wireless Communication (MAPWC-2010) in conjunction with Fifth International Conference on Broadband and Wireless Computing, Communication and Applications (BWCCA-2010), Nov. 2010, pp. 561-564.

[12] H. Maeda, Y. Zhang and H. Terashima, Numerical Analysis of Electromagnetic Wave Scattered by High-Contrast Dielectric Cylinder Using CIP Method, Proceedings of The 1st International Workshop on Information Networking and Wireless Communications (INWC 2011), in conjunction with The 14th International Conference on Network-Based Information Systems (NBiS 2011), Sep., 2011, pp. 491-496.

[13] A. Ishimaru, Electromagnetic Wave Propagation, Radiation, and Scattering, Chap. 11, Prentice-Hall International, 1991.

[14] H. Maeda, Numerical Technique for Electromagnetic Field Computation Including High Contrast Composite Material, as Chapter 3 of book entitled "Optical Communications", edited by Narottam Das, InTech Open Access Publisher, ISBN 978-953-51-0784-2, 2012, pp. 41-54.

H. Maeda was born in Fukuoka, Japan, in July 1966. He received the B.E. degree in electronics engineering, M.E. degree in information systems engineering and D.E. degree in computer science and communication engineering from Kyushu University in 1989, 1991, and 1996, respectively. In 1997, he joined to Department of Information and Communication Engineering in Fukuoka Institute of Technology, Japan. His research interest is experimental approach for electromagnetic band gap structure. $\mathrm{He}$ also studies numerical analysis technique for wave-guiding structure and nonlinear optics. Prof. Maeda is a member of Optical Society of America (OSA), Institute of Electronics, Information and Communication Engineers (IEICE) Japan and Japan Society of Applied Physics (JSAP).

Huili Chen was born in Jilin, China in 1988. She got bachelor's degree of information engineering from Fukuoka Institute of Technology in 2013. She currently studies in the graduate school of master's course for Communication and Information Networking. Her research topic is application of photonic crystal structure for signal processing. She is a student member of IEICE Japan.

Kazuya Tomiura was born in Nagasaki, Japan in 1990. He got bachelor's degree of information engineering from Fukuoka Institute of Technology in 2013. He currently studies in the graduate school of master's course for Communication and Information Networking. His research interest is design of periodic structure for signal processing by numerical method. He is a student member of IEICE Japan.

Kiyotoshi Yasumoto got the B.E., M.E., and D.E. degrees in communication engineering from Kyushu University, Fukuoka, Japan, in 1967, 1969, and 1977, respectively. In 1969, he joined the faculty of engineering of Kyushu University, where since 1988 he had been a professor of the Department of Computer Science and Communication Engineering. He was a visiting 
professor of University of Wisconsin in Madison in 1989 and a visiting fellow of Czechoslovakian Academy of Science in 1990. He has been a professor emeritus of Kyushu University since 2008. He is a fellow of IEICE Japan, Optical Society of America, the Chinese Institute of Electronics, respectively. He is also a senior member of IEEE AP, MTT, and LEOS Societies and a member of IEE Japan and the Electromagnetic Academy. He is currently a visiting researcher of the Computer Science Laboratory in Fukuoka Institute of Technology. His research interests are in electromagnetic wave theory, analytical and numerical techniques in microwave and photonics, and radiation and scattering in electron beam-plasma systems. He has served as a member of organizing, steering, technical program, and international advisory committees and as a session organizer for various international conferences. He has published more than 260 papers in various international journals and conference proceedings. 

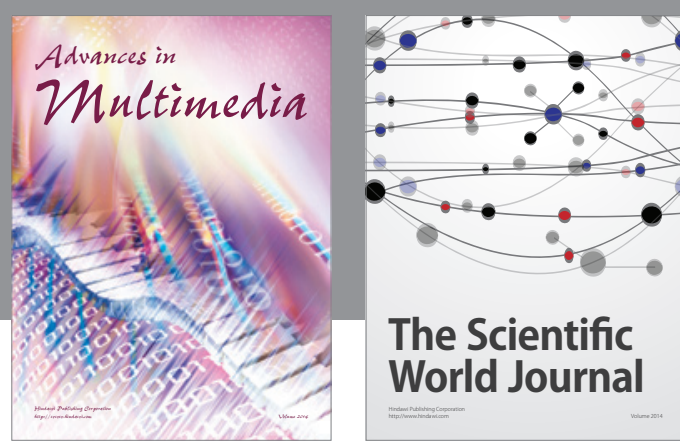

The Scientific World Journal
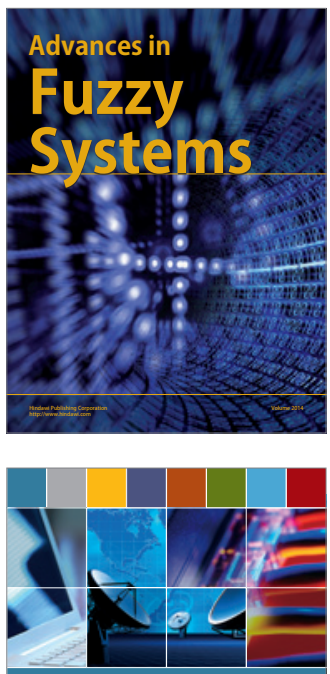

Computer Networks and Communications
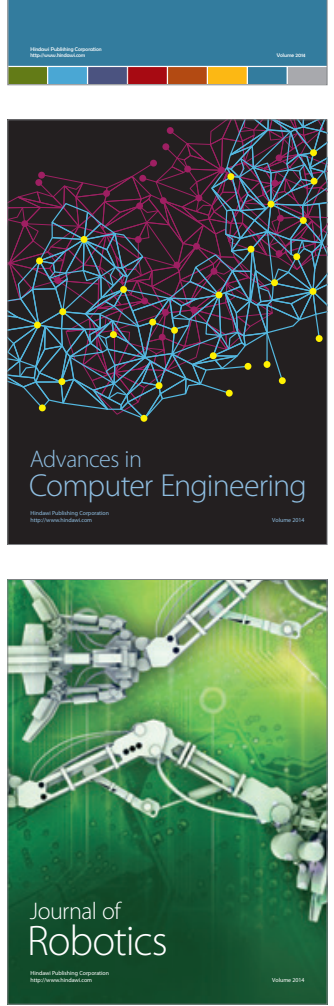
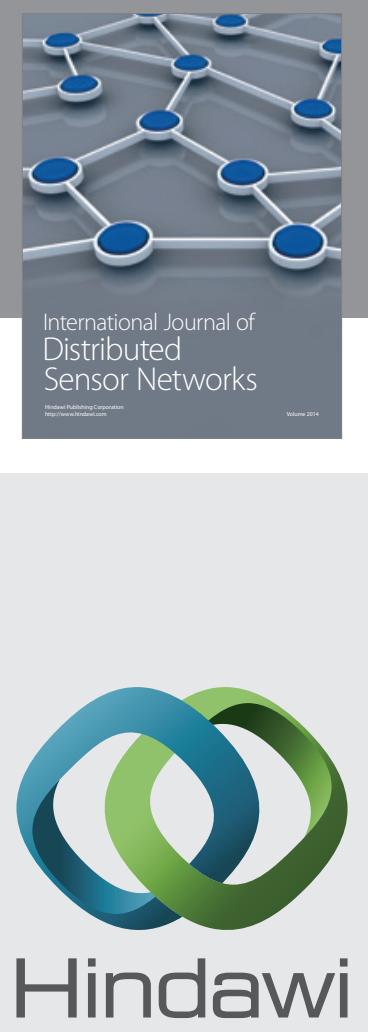

Submit your manuscripts at

http://www.hindawi.com
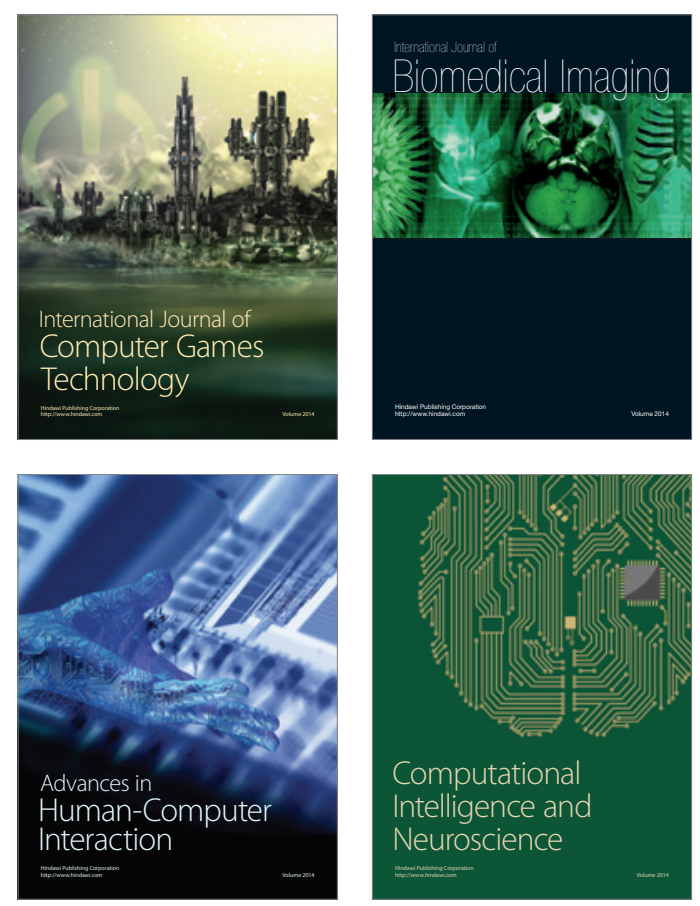
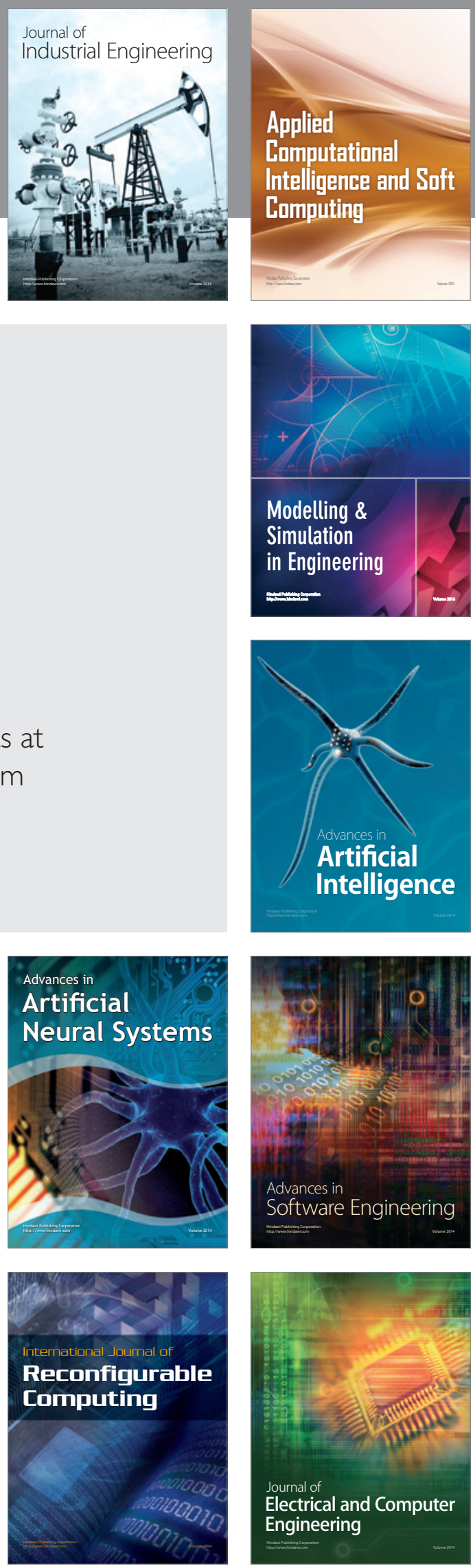